EGU21-15266

https://doi.org/10.5194/egusphere-egu21-15266

EGU General Assembly 2021

(c) Author(s) 2021. This work is distributed under

the Creative Commons Attribution 4.0 License.

\title{
Relation among geochemical elements in soil and red chicory as a tool for geographical origin identification.
}

\author{
Elena Marrocchino ${ }^{1}$, Serena Di Sarcina ${ }^{2}$, Carlo Ragazzi ${ }^{3}$, and Carmela Vaccaro ${ }^{1,4}$ \\ ${ }^{1}$ University of Ferrara, Department of Physics and Earth Science, via Saragat, 144121 Ferrara, Italy (mrrlne@unife.it) \\ ${ }^{2}$ University of Ferrara, Department of Department of Life Sciences and Biotechnology, via L. Borsari 46 44121, Ferrara, Italy \\ (serena.disarcina@edu.unife.it) \\ ${ }^{3}$ Consorzio Uomini di Massenzatica, via Indipendenza 39/a,44026 Massenzatica (Ferrara), Italy \\ (consorzio@uominidimassenzatica.it) \\ ${ }^{4}$ ISAC-CNR Institute of Atmospheric Sciences and Climate of the National Research Council of Italy, Via Piero Gobetti, 101, \\ 40129 Bologna, Italy (vcr@unife.it)
}

The identification of the geographical origin of food products is important for both consumers and producers to ensure quality and avoid label falsifications. Determination and authentication of the geographical origin of food products throughout scientific research have become recently relevant in investigations against frauds for consumer protection. Advances in methods and analytical techniques led to an increase in the application of fingerprinting analysis of foods for identification of geographical origin. Since in organic material the inorganic component is more stable than the organic one, several studies examined trace elements, suggesting the potential application for determination of geographical origin. Moreover, the studies on territoriality are based on the hypothesis that chemical elements detected in plants and in their products reflect those contained in the soil and, within these studies, the geographical features of the production area, such as the soil type and the climate, are considered relevant factors affecting the specific designation, so an accurate determination of geographical origin would be necessary to guarantee the quality and territoriality of the products.

In this light, two varieties of red chicory from the southern Po Delta area have been characterized together with the soil. The two inspected red chicory varieties (long-leaves and round-leaves) are cultivated in a well-defined area in the southern part of Po Delta, in an area sited around Massenzatica (Municipality of Mesola, Province of Ferrara, NE of Italy). Sampling was undertaken between October and December 2020 and samples were collected from a randomized field. Together with the red chicory also roots and soils have been collected in order to analyze each part and correlate the geochemical data obtained using ICP-MS and XRF techniques.

Purpose of this study is to establish a method to identify the geographical origin and the results confirm that some major and trace elements could be used as geochemical markers according to the geological areas. These elements, therefore, could be useful to establish geochemical fingerprints for testing the origin of this product and create a protected designation of origin label. 
